\title{
Liste des figures et des tableaux
}

\section{Figures}

Figure 1. - Processus schématique de maîtrise de la corrosion . . . . . . . 7

Figure 2. - Décollement et revêtement endommagé :

« Effet d'écran au courant de $\mathbf{P C}$ ». Possible corrosion . . . . . . . 7

Figure 3. - Corrosions sous décollement avec revêtement en bitume . . . . . 8

Figure 4. - Stockage de tubes $\ldots \ldots \ldots \ldots \ldots \ldots \ldots \ldots \ldots \ldots \ldots \ldots$

Figure 5. - Bardage de tubes ....................... 15

Figure 6. - Application d'un feutre anti roche $\ldots \ldots \ldots \ldots \ldots \ldots \ldots$

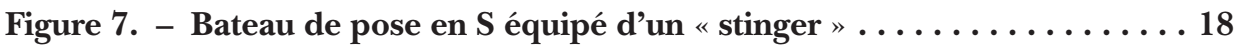

Figure 8. - Application par extrusion transversale (ou latérale) d'un 3LPE . 24

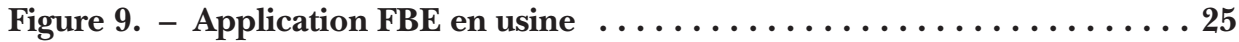

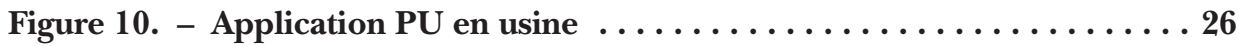

Figure 11. - Tube revêtu $3 \mathrm{LPP}+$ isolation thermique $\ldots \ldots \ldots \ldots \ldots$

Figure 12. - Epargne ou $\ll$ cut-back $» \ldots \ldots \ldots \ldots \ldots \ldots \ldots \ldots \ldots$

Figure 13. - Application de bande plastique à la machine semi-automatique

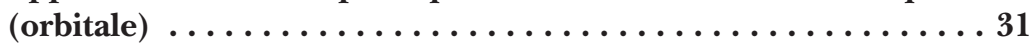

Figure 14. - Pose d'un manchon thermo-rétractable . . . . . . . . . 32

Figure 15. - Application de PP par projection à la flamme . . . . . . . 33

Figure 16. - Résine polyuréthanne appliquée au pistolet . . . . . . . . 34

Figure 17. - Application par projection d'époxy liquide .... . . . . 35

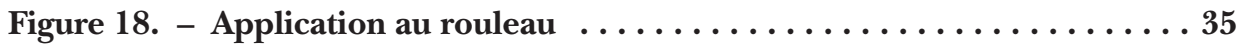

Figure 19. - Application de résine époxydique en poudre sur un joint de soudure ............................. 35

Figure 20. - Application de bandes de polyoléfine non-cristalline de basse

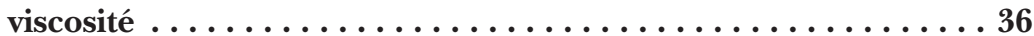


Figure 21. - Application de bande grasse (wax tape) . . . . . . . . . 38

Figure 22 - Application en cours de bande petrolatum après masticage . . . 38

Figure 23. - Application de cire de paraffine . . . . . . . . . . . 39

Figure 24. - Application de l'IMPP ................... 41

Figure 25. - Application de l'IMPU $\ldots \ldots \ldots \ldots \ldots \ldots \ldots \ldots \ldots \ldots \ldots$

Figure 26. - Principe de la protection cathodique $\ldots \ldots \ldots \ldots \ldots \ldots \ldots$

Figure 27. - Diagramme de Pourbaix du fer $\ldots \ldots \ldots \ldots \ldots \ldots \ldots$

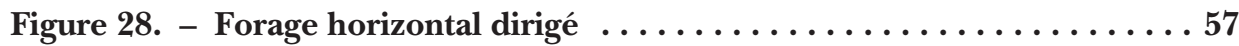

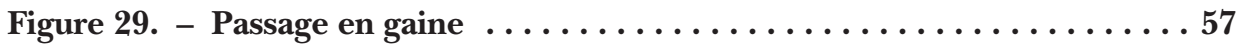

Figure 30. - Cuves revêtues en polyuréthanne $\ldots \ldots \ldots \ldots \ldots \ldots \ldots \ldots$

Figure 31. - Sortie de sol . . . . . . . . . . . . . . . . . . 62

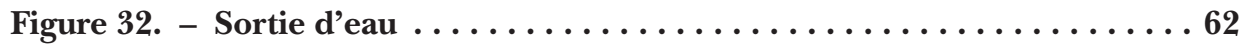

Figure 33. - Pièce de forme enrobée en bande grasse de cire microcristalline . . 63

Figure 34. - Mastic petrolatum en cours d'application ............ 64

\section{Tableaux}

Tableau 1. - Contraintes liées à la manutention, le transport, le stockage et le bardage, et propriétés des revêtements adaptés $\ldots \ldots \ldots 14$

Tableau 2. - Contraintes liées à la pose en milieu enterré et propriétés des revêtements adaptés $\ldots \ldots \ldots \ldots \ldots \ldots \ldots \ldots \ldots$

Tableau 3. - Contraintes liées à la pose en mer et propriétés des revêtements adaptés $\ldots \ldots \ldots \ldots \ldots \ldots \ldots \ldots \ldots$

Tableau 4. - Contraintes liées à la pose en mer et propriétés des revêtements adaptés $\ldots \ldots \ldots \ldots \ldots \ldots \ldots \ldots$

Tableau 5. - Contraintes liées à l'exploitation en milieu enterré et propriétés des revêtements adaptés . . . . . . . . . 19

Tableau 6. - Contraintes liées à l'exploitation en milieu marin et propriétés des revêtements adaptés . . . . . . . . . . . . 21

Tableau 7. - Contraintes liées la présence d'une protection cathodique et propriétés des revêtements adaptés $\ldots \ldots \ldots \ldots \ldots \ldots 22$

Tableau 8. - Systèmes de revêtements en fonction des paramètres d'exploitation de la canalisation $\ldots \ldots \ldots \ldots \ldots \ldots \ldots \ldots$ 
Tableau 9. - Descriptif des systèmes PE / PP appliqués par projection

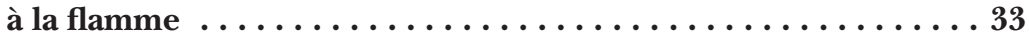

Tableau 10. - Revêtements usine applicables en fonction des conditions de service ......................... 56

Tableau 11. - Compatibilité des revêtements ............... 59 
